\title{
Application of the Adomian Decomposition Method (ADM) for Solving the Singular Fourth-Order Parabolic Partial Differential Equation
}

\author{
Béyi Boukary¹, Justin Loufouilou-Mouyedo², Joseph Bonazebi-Yindoula2 ${ }^{2}$, Gabriel Bissanga² \\ ${ }^{1}$ Universitéde Ouaga 1 Pr JKZ, Ouagadougou, Burkina Faso \\ ${ }^{2}$ Université Marien NGOUABI, Brazzaville, Republic of Congo \\ Email: *bonayindoula@yahoo.fr
}

How to cite this paper: Boukary, B., Loufouilou-Mouyedo, J., Bonazebi-Yindoula, J. and Bissanga, G. (2018) Application of the Adomian Decomposition Method (ADM) for Solving the Singular Fourth-Order Parabolic Partial Differential Equation. Journal of Applied Mathematics and Physics, 6, 1476-1480.

https://doi.org/10.4236/jamp.2018.67124

Received: March 23, 2018

Accepted: July 21, 2018

Published: July 24, 2018

Copyright $\odot 2018$ by authors and Scientific Research Publishing Inc. This work is licensed under the Creative Commons Attribution International License (CC BY 4.0).

http://creativecommons.org/licenses/by/4.0/

\begin{abstract}
In this paper, the ADM method is used to construct the solution of the singular fourth-order partial differential equation.
\end{abstract}

Keywords

SBA Method, Singular Fourth-Order Partial Differential Equation

\section{Introduction}

These last years, a lot of relatively new techniques as Adomian decomposition method (ADM), perturbation method, homotopy perturbation method, SOME BLAISE ABBO (SBA) method, variational iteration method etc. are used to solving a linear and nonlinear partial differential equations. Many problems are governed by partial differential equations, or by systems of partial differential equations. It is difficult to find their exact solutions. In this paper, we use the Adomian decomposition method (ADM) [1] [2] [3] [4] to find the exact solution of the singular fourth-order partial differential equation. This equation has been studied in [5], one used the homotopy perturbation to get the solution of the singular fourth-order partial differential equation in two space variables.

\section{About the ADM Method}

Suppose that we need to solve the following equation

$$
A u=f
$$


in a real Hilbert space $H$, where $A: H \rightarrow H$ is a linear or a nonlinear operator, $f \in H$ and $u$ is the unknown function. The principle of the ADM is based on the decomposition of the operator $A$ in the following form:

$$
A=L+R+N
$$

where $L+R$ is linear, $N$ nonlinear, $L$ invertible with $L^{-1}$ as inverse. Using that decomposition, equation ( 1 ) is equivalent to

$$
u=\theta+L^{-1} f-L^{-1} R u-L^{-1} N u
$$

where $\theta$ verifies $L \theta=0$. (3) is called the Adomian's fundamental equation or Adomian's canonical form. We look for the solution of (1) in a series expansion form $u=\sum_{n=0}^{+\infty} u_{n}$ and we consider $N u=\sum_{n=0}^{+\infty} A_{n}$ where $A_{n}$ are special polynomials of variables $u_{0}, u_{1}, \cdots, u_{n}$ called Adomian polynomials and defined by [1] [2] [3] [4]:

$$
A_{n}=\frac{1}{n !}\left[\frac{\mathrm{d}^{n}}{\mathrm{~d} \lambda^{n}} N\left(\sum_{i=0}^{+\infty} \lambda^{i} u_{i}\right)\right]_{\lambda=0}, \quad n=0,1,2, \cdots
$$

where $\lambda$ is a parameter used by "convenience". Thus (3) can be rewritted as follllows:

$$
\sum_{n=0}^{+\infty} u_{n}=\theta+L^{-1} f-L^{-1} R\left(\sum_{n=0}^{+\infty} u_{n}\right)-L^{-1}\left(\sum_{n=0}^{+\infty} A_{n}\right)
$$

We suppose that the series $\sum_{n=0}^{+\infty} u_{n}$ and $\sum_{n=0}^{+\infty} A_{n}$ are convergent, and obtain by identification the following Adomian algorithm:

$$
\left\{\begin{array}{l}
u_{0}=\theta+L^{-1} f \\
u_{1}=-L^{-1}\left(R u_{0}\right)-L^{-1} A_{0} \\
\quad \vdots \\
u_{n+1}=-L^{-1}\left(R u_{n}\right)-L^{-1} A_{n}, \quad n \geq 0
\end{array}\right.
$$

In practice it is often difficult to calculate all the terms of an Adomian series, so we approach the series solution by the truncated series:

$$
u=\sum_{i=0}^{n} u_{i}
$$

where the choice of $n$ depends on error requirements. If this series converges, the solution of (1) is:

$$
u=\lim _{n \rightarrow+\infty} \sum_{i=0}^{n} u_{i}
$$

\section{Resolution of the Singular Fourth-Order Parabolic Partial Differential Equation in $m$ Space Variables $(m \geq 2)$}

\subsection{The Singular Fourth-Order Parabolic Partial Differential Equation in Three Space Variables}

We consider the following singular fourth-order parabolic partial differential 
equation in three space variables:

$$
\begin{aligned}
& \frac{\partial^{2} u(x, y, z, t)}{\partial t^{2}}+\alpha\left(\frac{1}{x^{2}}+\frac{x^{4}}{6 !}\right) \frac{\partial^{4} u(x, y, z, t)}{\partial x^{4}}+\alpha\left(\frac{1}{y^{2}}+\frac{y^{4}}{6 !}\right) \frac{\partial^{4} u(x, y, z, t)}{\partial y^{4}} \\
& +\alpha\left(\frac{1}{z^{2}}+\frac{z^{4}}{6 !}\right) \frac{\partial^{4} u(x, y, z, t)}{\partial z^{4}}=0
\end{aligned}
$$

with the initial conditions

$$
\left\{\begin{array}{l}
u(x, y, z, t)=0 \\
\frac{\partial u(x, y, z, 0)}{\partial t}=\alpha+\frac{x^{6}}{6 !}+\frac{y^{6}}{6 !}+\frac{z^{6}}{6 !}
\end{array}\right.
$$

From (8), we have:

$$
\begin{aligned}
u(x, y, z, t)= & \left(\alpha+\frac{x^{6}}{6 !}+\frac{y^{6}}{6 !}+\frac{z^{6}}{6 !}\right) t-\alpha\left(\frac{1}{x^{2}}+\frac{x^{4}}{6 !}\right) \int_{0}^{t} \int_{0}^{h} \frac{\partial^{4} u(x, y, z, s)}{\partial x^{4}} \mathrm{~d} s \mathrm{~d} h \\
& -\alpha\left(\frac{1}{y^{2}}+\frac{y^{4}}{6 !}\right) \int_{0}^{t} \int_{0}^{h} \frac{\partial^{4} u(x, y, z, s)}{\partial y^{4}} \mathrm{~d} s \mathrm{~d} h \\
& -\alpha\left(\frac{1}{z^{2}}+\frac{z^{4}}{6 !}\right) \int_{0}^{t} \int_{0}^{h} \frac{\partial^{4} u(x, y, z, s)}{\partial z^{4}} \mathrm{~d} s \mathrm{~d} h
\end{aligned}
$$

We suppose that the solution of (8) has the following form:

$$
u(x, y, z, t)=\sum_{n=0}^{\infty} u_{n}(x, y, z, t)
$$

From (10) and (11) we have:

$$
\begin{aligned}
\sum_{n=0}^{\infty} u_{n}(x, y, z, t) & \left(\alpha+\frac{x^{6}}{6 !}+\frac{y^{6}}{6 !}+\frac{z^{6}}{6 !}\right) t-\alpha\left(\frac{1}{x^{2}}+\frac{x^{4}}{6 !}\right) \sum_{n=0}^{\infty} \int_{0}^{t} \int_{0}^{h} \frac{\partial^{4} u_{n}(x, y, z, s)}{\partial x^{4}} \mathrm{~d} s \mathrm{~d} h \\
& -\alpha\left(\frac{1}{y^{2}}+\frac{y^{4}}{6 !}\right) \sum_{n=0}^{\infty} \int_{0}^{t} \int_{0}^{h} \frac{\partial^{4} u_{n}(x, y, z, s)}{\partial y^{4}} \mathrm{~d} s \mathrm{~d} h \\
& -\alpha\left(\frac{1}{z^{2}}+\frac{z^{4}}{6 !}\right) \sum_{n=0}^{\infty} \int_{0}^{t} \int_{0}^{h} \frac{\partial^{4} u_{n}(x, y, z, s)}{\partial z^{4}} \mathrm{~d} s \mathrm{~d} h
\end{aligned}
$$

From (12), we obtain the following Adomian algorithm:

$$
\left\{\begin{aligned}
u_{0}(x, y, z, t)= & \left.\alpha+\frac{x^{6}}{6 !}+\frac{y^{6}}{6 !}+\frac{z^{6}}{6 !}\right) t=\left(3+\frac{x^{6}}{6 !}+\frac{y^{6}}{6 !}+\frac{z^{6}}{6 !}\right) t+(\alpha-3) t \\
u_{n+1}(x, y, z, t)= & -\alpha\left(\frac{1}{x^{2}}+\frac{x^{4}}{6 !}\right) \sum_{n=0}^{\infty} \int_{0}^{t} \int_{0}^{h} \frac{\partial^{4} u_{n}(x, y, z, s)}{\partial x^{4}} \mathrm{~d} s \mathrm{~d} h \\
& -\alpha\left(\frac{1}{y^{2}}+\frac{y^{4}}{6 !}\right) \sum_{n=0}^{\infty} \int_{0}^{t} \int_{0}^{h} \frac{\partial^{4} u_{n}(x, y, z, s)}{\partial y^{4}} \mathrm{~d} s \mathrm{~d} h \\
& -\alpha\left(\frac{1}{z^{2}}+\frac{z^{4}}{6 !}\right) \sum_{n=0}^{\infty} \int_{0}^{t} \int_{0}^{h} \frac{\partial^{4} u_{n}(x, y, z, s)}{\partial z^{4}} \mathrm{~d} s \mathrm{~d} h, n \geq 0
\end{aligned}\right.
$$

From (13), we obtain: 


$$
\left\{\begin{aligned}
u_{0}(x, y, z, t) & =\left(3+\frac{x^{6}}{6 !}+\frac{y^{6}}{6 !}+\frac{z^{6}}{6 !}\right) t+(\alpha-3) t \\
u_{1}(x, y, z, t) & =-\frac{\alpha}{2}\left(3+\frac{x^{6}}{6 !}+\frac{y^{6}}{6 !}+\frac{z^{6}}{6 !}\right) \frac{t^{3}}{3 !} \\
u_{2}(x, y, z, t) & =\frac{\alpha^{2}}{4}\left(3+\frac{x^{6}}{6 !}+\frac{y^{6}}{6 !}+\frac{z^{6}}{6 !}\right) \frac{t^{3}}{5 !} \\
u_{3}(x, y, z, t) & =-\frac{\alpha^{3}}{8}\left(3+\frac{x^{6}}{6 !}+\frac{y^{6}}{6 !}+\frac{z^{6}}{6 !}\right) \frac{t^{3}}{7 !} \\
\vdots & \\
u_{n}(x, y, z, t) & =(-1)^{n} \sqrt{\frac{2}{\alpha}} \frac{\left(t \sqrt{\frac{\alpha}{2}}\right)^{2 n+1}}{(2 n+1) !}\left(3+\frac{x^{6}}{6 !}+\frac{y^{6}}{6 !}+\frac{z^{6}}{6 !}\right), n \geq 1
\end{aligned}\right.
$$

Thus

$$
\begin{aligned}
u(x, y, z, t)= & \sum_{n=0}^{\infty} u_{n}(x, y, z, t)=\left(3+\frac{x^{6}}{6 !}+\frac{y^{6}}{6 !}+\frac{z^{6}}{6 !}\right) t+(\alpha-3) t \\
& +\sqrt{\frac{2}{\alpha}}\left(3+\frac{x^{6}}{6 !}+\frac{y^{6}}{6 !}+\frac{z^{6}}{6 !}\right) \sum_{n=0}^{\infty}(-1)^{n} \frac{\left(t \sqrt{\frac{\alpha}{2}}\right)^{2 n+1}}{(2 n+1) !} \\
u(x, y, z, t)= & (\alpha-3) t+\sqrt{\frac{2}{\alpha}}\left(3+\frac{x^{6}}{6 !}+\frac{y^{6}}{6 !}+\frac{z^{6}}{6 !}\right) \sin t \sqrt{\frac{\alpha}{2}}
\end{aligned}
$$

Remark: In the case of the singular fourth-order parabolic partial differential equation in two space variables, we have:

$$
u(x, y, t)=(\alpha-2) t+\sqrt{\frac{2}{\alpha}}\left(2+\frac{x^{6}}{6 !}+\frac{y^{6}}{6 !}\right) \sin t \sqrt{\frac{\alpha}{2}}
$$

and we recover the examined case in [5], where $\alpha=2$,

$$
u(x, y, t)=\left(2+\frac{x^{6}}{6 !}+\frac{y^{6}}{6 !}\right) \sin t
$$

\subsection{Main Result}

\section{Proposition}

The exact solution of the following singular fourth-order parabolic partial differential equation in $m$ space variables $m \in \mathbb{N},(m \geq 2)$ :

$$
\frac{\partial^{2} u\left(x_{1}, \cdots, x_{m}, t\right)}{\partial t^{2}}+\alpha \sum_{j=1}^{m}\left(\frac{1}{x_{j}^{2}}+\frac{x_{j}^{4}}{6 !}\right) \frac{\partial^{4} u\left(x_{1}, \cdots, x_{m}, t\right)}{\partial x_{j}^{4}}=0, \alpha \in \mathbb{R}_{+}^{*}
$$

with the following initial conditions

$$
\left\{\begin{array}{l}
u\left(x_{1}, \cdots, x_{m}, 0\right)=0 \\
\frac{\partial u\left(x_{1}, \cdots, x_{m}, 0\right)}{\partial t}=\alpha+\sum_{j=1}^{m} \frac{x_{j}^{6}}{6 !}
\end{array}\right.
$$


is:

$$
u\left(x_{1}, \cdots, x_{m}, t\right)=(\alpha-m) t+\left(m+\sum_{j=1}^{m} \frac{x_{j}^{6}}{6 !}\right) \sqrt{\frac{2}{\alpha}} \sin t \sqrt{\frac{\alpha}{2}}
$$

\section{Proof}

If $t=0$, from (20), we have $u\left(x_{1}, \cdots, x_{m}, 0\right)=0$.

From (20), we get:

$$
\frac{\partial u\left(x_{1}, \cdots, x_{m}, t\right)}{\partial t}=(\alpha-m)+\left(m+\sum_{j=1}^{m} \frac{x_{j}^{6}}{6 !}\right) \cos t \sqrt{\frac{\alpha}{2}}
$$

and if $t=0$, we have: $\frac{\partial u\left(x_{1}, \cdots, x_{m}, 0\right)}{\partial t}=\alpha+\sum_{j=1}^{m} \frac{x_{j}^{6}}{6 !}$

$$
\begin{gathered}
\frac{\partial^{2} u\left(x_{1}, \cdots, x_{m}, t\right)}{\partial t^{2}}=-\sqrt{\frac{\alpha}{2}}\left(m+\sum_{j=1}^{m} \frac{x_{j}^{6}}{6 !}\right) \sin t \sqrt{\frac{\alpha}{2}} \\
\frac{\partial^{4} u\left(x_{1}, \cdots, x_{m}, t\right)}{\partial x_{j}^{4}}=\sqrt{\frac{2}{\alpha}} \frac{x_{j}^{2}}{2 !} \sin t \sqrt{\frac{\alpha}{2}} \\
\left(\frac{1}{x_{j}^{2}}+\frac{x_{j}^{4}}{6 !}\right) \frac{\partial^{4} u}{\partial x_{j}^{4}}=\sqrt{\frac{2}{\alpha}} \frac{x_{j}^{2}}{2 !}\left(\frac{1}{x_{j}^{2}}+\frac{x_{j}^{4}}{6 !}\right) \sin t \sqrt{\frac{\alpha}{2}}=\frac{1}{2 !}\left(1+\frac{x_{j}^{6}}{6 !}\right) \sqrt{\frac{2}{\alpha}} \sin t \sqrt{\frac{\alpha}{2}} \\
\alpha \sum_{j=1}^{m}\left(\frac{1}{x_{j}^{2}}+\frac{x_{j}^{4}}{6 !}\right) \frac{\partial^{4} u}{\partial x_{j}^{4}}=\frac{\alpha}{2}\left(m+\sum_{j=1}^{m} \frac{x_{j}^{6}}{6 !}\right) \sqrt{\frac{2}{\alpha}} \sin t \sqrt{\frac{\alpha}{2}}
\end{gathered}
$$

From (22) and (25), we obtain:

$$
\frac{\partial^{2} u}{\partial t^{2}}+\alpha \sum_{j=1}^{m}\left(\frac{1}{x_{j}^{2}}+\frac{x_{j}^{4}}{6 !}\right) \frac{\partial^{4} u}{\partial x_{j}^{4}}=0
$$

\section{Conclusion}

Through this example, we showed again the usefulness of the Adomian decomposition method, in the search of an approximate solution of a linear or nonlinear equation; and this method gives us the exact solution.

\section{References}

[1] Abbaoui, K. (1995) Les fondements de la méthode décompositionnelle d'Adomian et application à la résolution de problèmes issus de la biologie et de la médécine. Thèse de doctorat de l'Université Paris VI.

[2] Abbaoui, K. and Cherruault, Y. (1994) Convergence of Adomian Method Applied to Differential Equations. Mathematical and Computer Modelling, 28, 103-109.

[3] Abbaoui, K. and Cherruault, Y. (1994) Convergence of Adomian Method Applied to Non Linear Equations. Mathematical and Computer Modelling, 20, 60-73. https://doi.org/10.1016/0895-7177(94)00163-4

[4] Abbaoui, K. and Cherruault, Y. (1999) The Decomposition Method Applied to the Cauchy Problem. Kybernetes, 28, 68-74. https://doi.org/10.1108/03684929910253261

[5] Mohyud-Dinand, S.T. and Noor, M.A. (2009) Homotopy Perturbation Method for Solving Partial Differential Equations. Z. Naturforsch, 64a, 157-170. 